\title{
A Low Energy Clock Network with a Huge Number of Local Synchronized Oscillators
}

This paper was downloaded from TechRxiv (https://www.techrxiv.org).

\section{LICENSE}

CC BY-NC-SA 4.0

SUBMISSION DATE / POSTED DATE

$28-11-2021 / 06-12-2021$

\section{CITATION}

Carlstedt, Gunnar; Rimborg, Mats (2021): A Low Energy Clock Network with a Huge Number of Local Synchronized Oscillators. TechRxiv. Preprint. https://doi.org/10.36227/techrxiv.17088812.v1

DOI

10.36227/techrxiv.17088812.v1 


\title{
A Low Energy Clock Network with a Huge Number of Local Synchronized Oscillators
}

\author{
Gunnar Carlstedt and Mats Rimborg
}

\begin{abstract}
A clock system for a huge grid of small clock regions is presented. There is an oscillator in each clock region, which drives the local clock of a processing element (PE). The oscillators are kept synchronized by exploiting the phase of their neighbors. In an infinite mesh, the clock skew would be zero, but in a network of limited size there will be fringe effects. In a mesh with $25 \times 25$ oscillators, the maximum skew between neighboring regions is within $3.3 \mathrm{ps}$. By slightly adjusting the free running frequency of the oscillators, this skew can be reduced to $1.2 \mathrm{ps}$. The mesh may contain millions of clock regions.

Because there is no central clock, both power consumption and clock frequency can be improved compared to a conventional clock distribution network. A PE of $150 \times 150 \mu \mathrm{m}^{2}$ running at 6.7 GHz with 93 master-slave flip-flops is used as an example. The PE-internal clock skew is less than $2.3 \mathrm{ps,}$ and the energy consumption of the clock system $807 \mu \mathrm{W}$ per PE. It corresponds to an effective gate and wire capacitance of $509 \mathrm{aF}$, or 7.3 gate capacitances.

Power noise is reduced by scheduling the local oscillators gradually along one of the grid's axes. In this way, surge currents, which generally have their peaks at the clock edges, are distributed evenly over a full clock cycle.
\end{abstract}

Index Terms - array of processing elements, clock, clock skew, power noise, timing

\section{INTRODUCTION}

$\mathrm{M}$ anycore processors are designed for a high degree of parallel processing with thousands, or even more, simple independent cores. One such new and emerging computer architecture is the Surface Based Processor (SBP) [1]. It has an orthogonal package switching network ( NoC), where the links are in the 100-200 $\mu \mathrm{m}$ range. Within each square of links, there is a Processing Element (PE). The PEs are tightly connected, and all communication between the PEs traverse the links at a frequency in the $10 \mathrm{GHz}$ range. The link endpoints have to be in phase with the PE clocks, which necessitates a low clock skew.

The SBP architecture differs from conventional CPUs, GPUs, and DSPs in its magnitude of PEs, the tight connection between these, and their speed. The number of PEs can be

This paragraph of the first footnote will contain the date on which you submitted your paper for review.

The authors are with the Department of Computer Science and Engineering, Chalmers University of Technology, SE-412 96 Göteborg, Sweden (e-mail: gunnar@carlstedt.se and mats@rimborg.se). from about a thousand up to millions. The communication bandwidth is in the range of Petabits/s $(1.5 \mathrm{~Pb} / \mathrm{s}$ for 100,000 PEs). Metastable states would create noticeable errors in asynchronous data streams and cause failures. Therefore, all PEs should be synchronized. From each PE's point of view, only the four neighboring PEs whose nodes have direct contact horizontally and vertically need to have low skew, but since these in their turn also have neighbors this condition is recursive. Each NoC node keeps its clock in phase with the neighbors' clocks.

This article covers the clock system of the entire processor. The work has been motivated by the need for an optimal SBP clock system, but the design is in no way limited to this application.

There are basically two ways to create clocks:

1. One source oscillator provides clock pulses that are distributed to all PEs and provide the pace. Most commonly, electronic devices use meshes, a hierarchical H-tree structure [2], or a combination thereof to distribute the central clock.

2. Each PE has a locally powered oscillator that only sends full swing clock pulses to sinks within the PE. The synchronization is made by observing its neighbors. Like in a giant parade, soldiers (oscillators) are marching in pace at a nominal speed. The synchronization is accurate enough to visually give the impression of all legs moving as one. The distance between the soldiers is constant and the speed of their legs is controlled by the soldiers' observations of their neighbors. This is the principle of the clock system described here.

In this clocking paradigm, the phase is controlled by keeping the resonators of neighboring oscillators tightly coupled. The aggregate sinusoidal signal is summed up on a wire (RC) network. The signal level on this network may be low, depending on the amplification in the oscillator. The power consumption of the synchronization is proportional to the signal amplitude. The process variations are assumed to be small within each PE. Neighboring clocks are also within the same limited range. Therefore, the influence from global process variations is low.

A clock system inevitably generates power noise. Within each PE, the clock pulses should be synchronous to maximize performance. However, the clocks can be slightly out of phase compared to the nearby PEs, to even out the power noise from 
individual PEs. Revisiting the comparison with a military parade, it corresponds to letting each line of soldiers lag slightly when crossing a suspension bridge to prevent it from breaking. The phase is distributed over an entire cycle in the long parade, and the marching beats are evened out.

The contribution of this work is:

- Local oscillators synchronized by a single wire mesh.

- A high frequency clock system with low energy consumption.

- Reduced power noise by evening out current surges.

The mesh with oscillators is described in section II. The pulse shaper that converts the sinusoidal oscillator signal to an internal square wave clock of the PE is described in section III. The PE clock with buffer and internal clock distribution is described in section $I V$.

The clock system is evaluated in the following sections. This is done by using details from an SBP design. The clock period is $150 \mathrm{ps}$, power supply voltage $0.8 \mathrm{~V}$, and PE size $150 \times 150 \mu \mathrm{m}$ implemented in a typical $14 \mathrm{~nm}$ technology. The sinusoidal synchronization signal has an amplitude of $200 \mathrm{mV}$.

An infinite area of oscillators would have them all fully in phase. The use of a limited number of oscillators is evaluated in section $V$. The influences from process variations are considered in section VI. The reduction of power noise by scheduling PEs is described in section VII.

Finally, section VIII describes some relevant related work, and section $I X$ contains our conclusion.

\section{Mesh With Oscillators}

The mechanism is a mesh with oscillators, see Fig. 1, where each link is a single wire. The transmission lines are modeled with an inductance free lossy representation. Transmission lines with lowest loss can be used, around $2.5 \mathrm{ohm} / \mu \mathrm{m}$ and $150 \mathrm{aF} / \mu \mathrm{m}$.

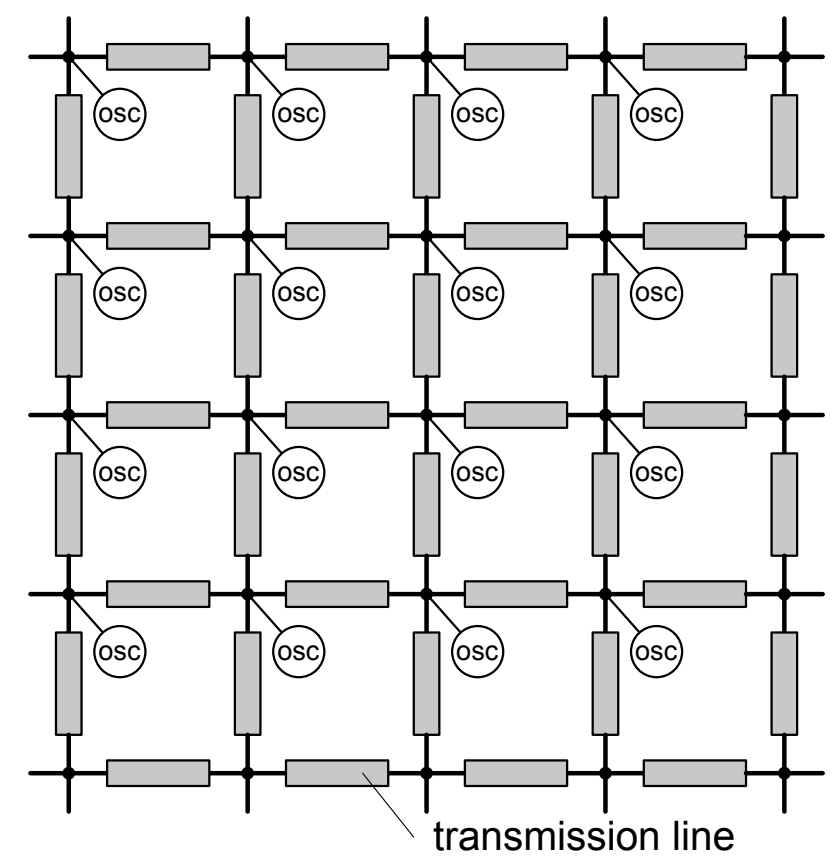

Fig. 1. A mesh of oscillators.
An ordinary square wave clock signal would be heavily distorted at high frequencies. A sinusoidal signal is simply attenuated and phase shifted, but the waveform remains intact. If all nodes have a corresponding oscillator, the signal in one node is the sum of the attenuated outputs from all nodes. For these reasons, a sine wave is used on the global mesh. In an infinite structure, all oscillators will have the same signal. In a network of limited sized, there will be fringe effects.

All oscillators are of the same kind, and locally powered. They are strongly coupled with the mesh as a common resonator used for synchronization and will have the same amplitude and phase. This large number of parallel oscillators act together with a heavily conducting negative resistance and resemble a rotary travelling-wave oscillator [3].

A low clock voltage will keep the power consumption low, and the synchronization will keep the clock skew close to zero. This implementation can be used on a chip of arbitrary size with a huge number of PEs.

\section{A. Local Oscillator}

The local oscillator is based on an ordinary ring oscillator that consists of a loop with an odd number of inverters. A minimal oscillator running at full swing with three inverters M1-M6, without load and Vdd $=0.8 \mathrm{~V}$, runs at approximately $104 \mathrm{GHz}$, see schematics in Fig. 2. At $0.27 \mathrm{~V}$ the oscillator runs at $22.7 \mathrm{GHz}$. Such an oscillator will need stepwise increasing pairs of 1,5 , and 24 parallel FinFETs to achieve required driving capability for $300 \mu \mathrm{m}$ wires with a capacitance of $45 \mathrm{fF}$. It runs slower due to high network capacitance and consumes $80 \mu \mathrm{A}$ at $6.3 \mathrm{GHz}$.

By utilizing the network as a common resonant circuit, all oscillators will run in parallel, and be synchronized without any additional phase locking components. The power dissipation is distributed over the mesh area. The same power voltage $V_{\text {osc }}$ is used for all oscillators, and its level also controls the frequency. It may be managed by an external PLL.

\section{PUlse Shaping}

The sine wave $V_{\text {synch }}$ is reshaped into pulses in the circuit M7-M10, see Fig. 2, and fed to an inverter. It consists of an nchannel transistor M10 that has a threshold voltage $V_{T}$. Its drain current $I d M 10$ has a quadratic relationship to the input voltage. An identical $\mathrm{n}$-channel transistor M8 creates a corresponding current $I d M 8$, but relative to an upper threshold $V_{o s c}-V_{T}$. Transistors M7 and M9 constitute a current mirror. The drain current IdM9 equals $I d M 8$. The inverter output $O S C b$ switches when the drain currents $I d M 9$ and $I d M 10$ are equal, which occurs when $V_{\text {synch }}$ is at half the $V_{\text {osc }}$ voltage. A second inverter follows, which increases the slew rate.

$$
\begin{aligned}
& I d M 10=k \times\left(V_{\text {synch }}-V_{T}\right)^{2} \\
& \begin{array}{l}
I d M 9=I d M 8=k \times\left(V_{\text {osc }}-V_{\text {synch }}-V_{T}\right)^{2} \\
\text { OSC } \boldsymbol{B}=I d M 10>I d M 9= \\
\quad=k \times\left(V_{\text {synch }}-V_{T}\right)^{2}>k \times\left(V_{\text {osc }}-V_{\text {synch }}-V_{T}\right)^{2}= \\
\quad=V_{\text {synch }}-V_{T}>V_{\text {osc }}-V_{\text {synch }}-V_{T}= \\
\quad=2 \times V_{\text {synch }}>V_{\text {osc }}
\end{array}
\end{aligned}
$$


The current mirror is used to convert the low swing voltage to a full digital voltage level, and the threshold voltage depends on the scaling of the transistors. The n-channel transistors and $\mathrm{p}$-channel transistors are two matching pairs $(M 7+M 9$ and $M 8+M 10)$, which are placed next to each other. Therefore, their transfer characteristics are almost identical. At low input frequency, the threshold is stable at half of $V_{\text {osc. }}$. At high frequency, capacitances move the threshold voltage somewhat, but less than $20 \mathrm{mV}$. There is a propagation delay of $18 \mathrm{ps}$ in the pulse shaper, according to Spice simulations.

\section{PE ClOCK}

The PE of a Surface Based Processor is used to describe the local clock distribution. It is characterized by high frequency and small dimensions.

The clock system except for the oscillator and the pulse shaper consumes $903 \mu \mathrm{A}$ at $0.8 \mathrm{~V}$ with a $150 \mathrm{ps}$ clock cycle. The oscillator and the pulse shaper contribute with another $80+27=107 \mu \mathrm{A}$. This makes a total of $807 \mu \mathrm{W}$ for the entire clock system. About a quarter of the energy is lost in the global clock wire $C L K$ and another quarter in the secondary clock wires. The remaining half is consumed in the transistors.

The schematics of the clock system of a Surface Based Processor is shown in Fig. 3. It consists of an oscillator, a pulse shaper, a gate for the clock, and a clock buffer driving a central clock wire $C L K$. The clock wire is equipped with buffers to control the master-slave registers of the PE.

\section{A. Structure}

A preliminary SBP PE design has 93 master-slave registers. They consist of $4 \times 93$ transmission gates with 372 n-channel and $372 \mathrm{p}$-channel transistors, with a gate capacitance of $70 \mathrm{aF}$ each. The total fan-out is 744 , i.e., a capacitance of $52 \mathrm{fF}$. The floorplan is determined by energy consumption and wire density restrictions. Therefore, the registers are placed in a Tshaped structure. A single-phase clock would implement this clock distribution as a tiny wire with minimal capacitance. However, connecting all clocks to the wire as a fishbone would overload the wire and cause prohibitive propagation delays. Therefore, a two-level hierarchy is used, where each memory block and register structure uses one connection.

The arms of the T-shaped clock have about the same length, about $250 \mu \mathrm{m}$ altogether, with a total capacitance of $38 \mathrm{fF}$. The central clock buffer could be split into three smaller

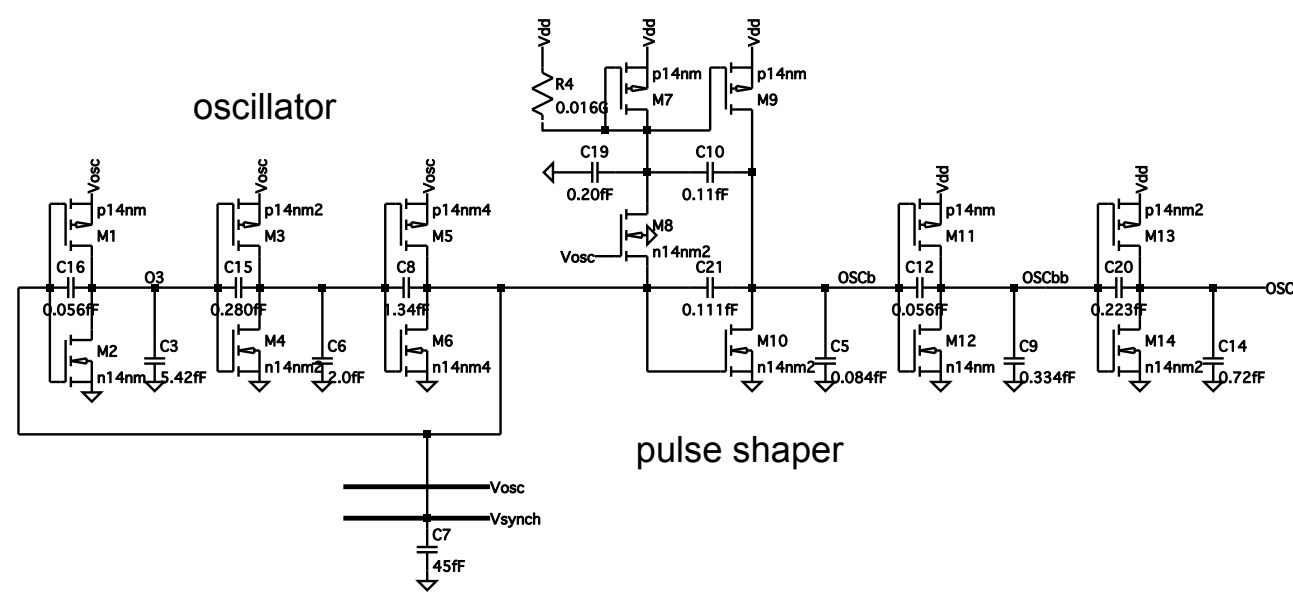

\section{B. Clock Buffer}

buffers, but there is no gain in this since it may cause additional skew from process variations. All surge currents would still come from the same area.

There are 19 secondary buffers, which consist of an input inverter followed by a second inverter that generates steep pulses with rise and fall times of 7 ps. They drive two output stages with a considerable load. Resulting from the high input slew rate there are very small short circuit currents, see Fig. 4. The aggregated output stages drive secondary clock wires with a total capacitance of $83 \mathrm{fF}$ for wires and transistors.

The clock buffer uses transistors $M b 1-M b 18$, see Fig. 5. Current surges are very large, around $5 \mathrm{~mA}$. To provide this current, a single FinFET is insufficient. High current FETs are implemented by duplicating ordinary transistors and connecting them in parallel. The last output stage needs about 60 FinFETs for each n- and p-channel transistor, $M b 13$ and $M b 14$, see Fig. 6b. They could be placed under the $C L K$ wire. The connection is a short stub from each transistor.

An ordinary inverter would have a high short circuit current. Therefore, the buffer is instead designed with a minimal inverter, consisting of only one $\mathrm{n}$ - and one p-channel FinFET, $M b 1$ and $M b 2$ (marked in black). These are only used at very low frequency for testing purpose. To this, two surge drivers are added (marked in red), for rising and falling edges. The rising edge surge driver consists of the transistors $M b 4$, $M b 10$ and $M b 13$, implemented as $1+5+60$ parallel transistors. Because transistor $M b 3$ is only conducting when $C L K$ is low, the surge is performed only at the beginning of a rising clock edge. The inverter $M b 15-M b 16$ detects when $C L K$ has switched across the midpoint. An additional load capacitance $C b 9$ causes a slight delay until $C L K$ has reached almost full swing. Transistors $M b 5$ and $M b 9$ precharge the switching nodes and stop the driving. Mb17-Mb18 will invert the polarity. The switching is shown in Fig. 6a. There is no short circuit current in the output node, and it is negligible in all the other nodes, see Fig. 6b-Fig. 6d.

\section{Clock Wire}

The $C L K$ wire is one of many parallel wires implemented in modern $3 \mathrm{D}$ technology. The wire is a standing balk with low- $\kappa$ dielectric on each side. The balks act as plate capacitors. The major wire capacitance is to the two adjacent wires $V d d$ and $G N D$, to reduce cross-talk.

Fig. 2. Schematics of the oscillator with pulse shaping circuit. The circuit can be optimized further. 


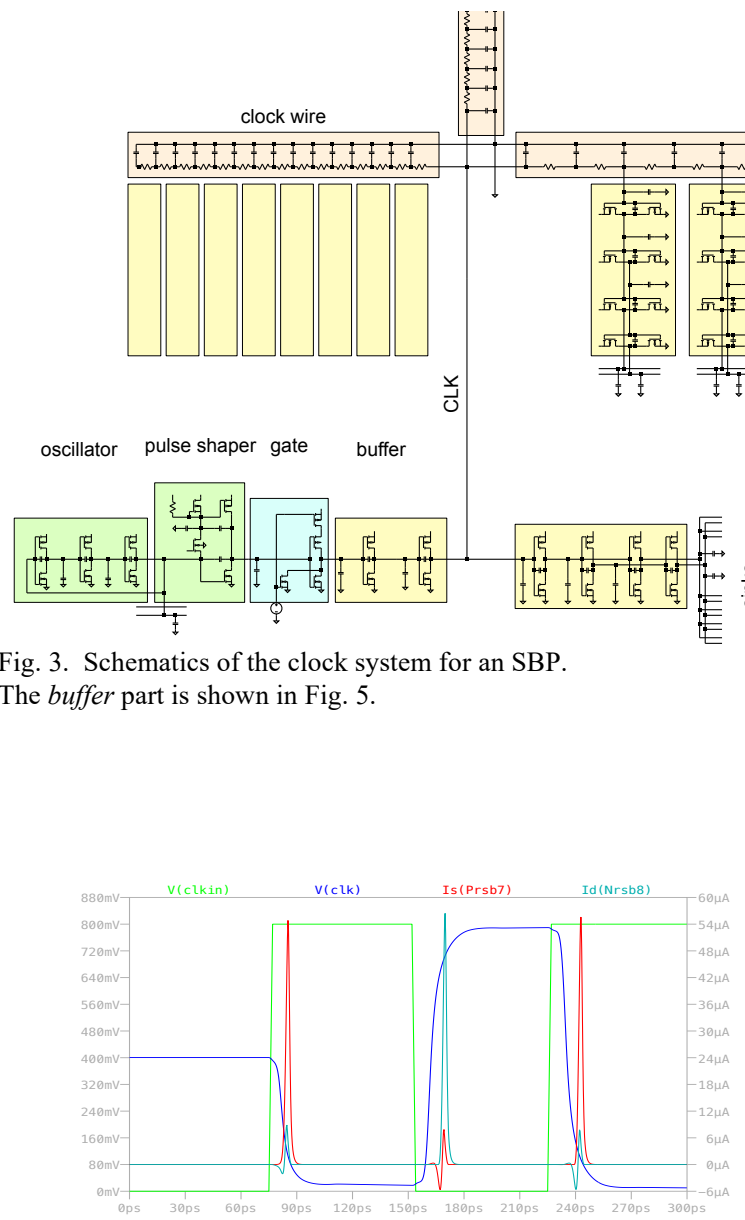

Fig. 4. Surge currents in the secondary buffers.

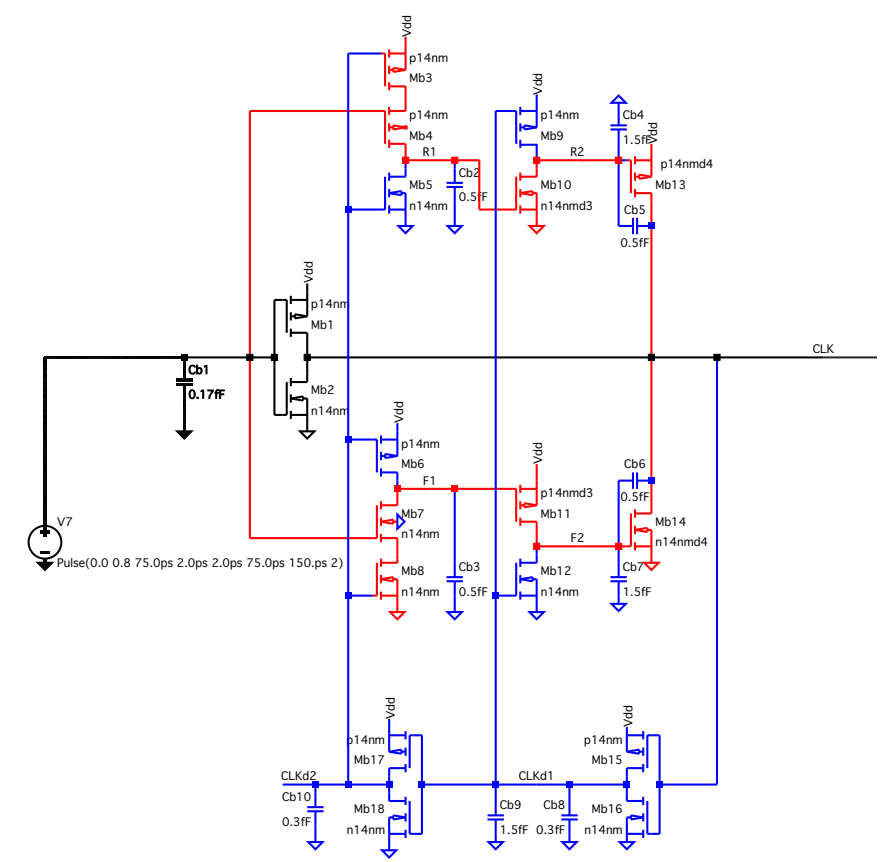

Fig. 5. Schematics of the clock buffer.

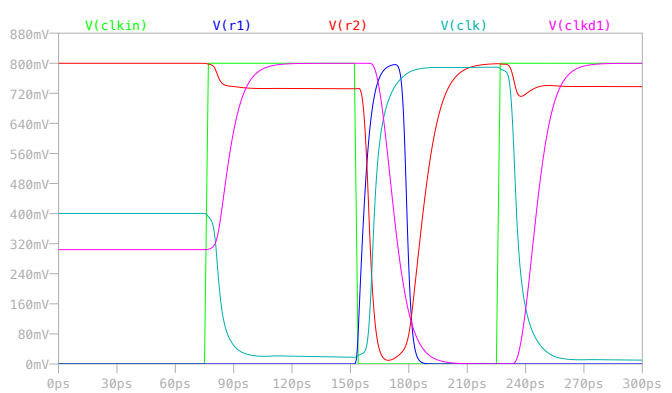

Fig. 6a. Clock buffer voltages.

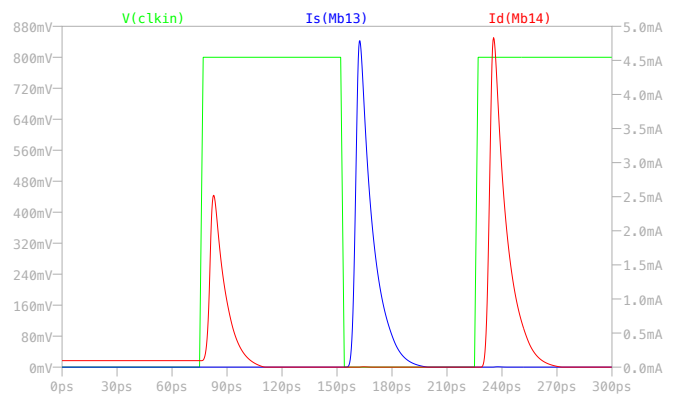

Fig. 6b. Clock buffer Mb13 and Mb14 currents.

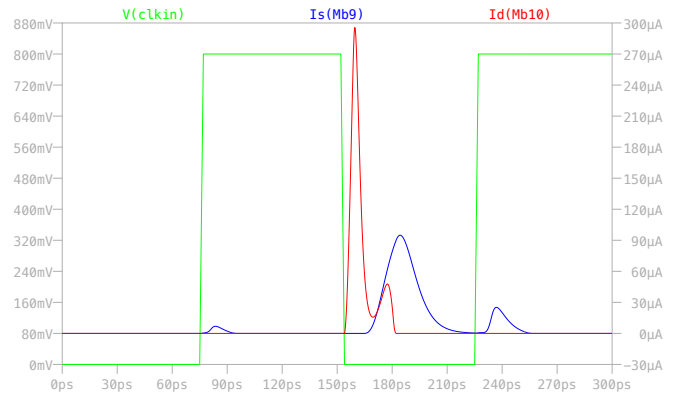

Fig. 6c. Clock buffer Mb9 and Mb10 currents.

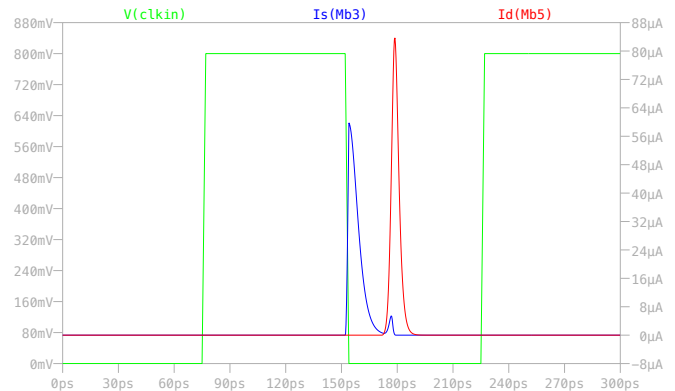

Fig. 6d. Clock buffer Mb3 and Mb5 currents. 


\section{Mesh Clock SKeW}

As stated in section II, an infinite area of clocks would have no clock skew between any pair of oscillators. This assumption has been tested with a linear structure instead of an array. Two loops of oscillators separated by transmission lines were simulated, one with 25 and the other with 64 oscillators. In these initial simulations intended to verify the theoretical assumption, all clocks and wires were identical. No skew was detected in any of the cases, see Fig. 7. The lower part is a magnification of a slope.

When the oscillators are placed along a vector, their environments differ. In the middle of the vector, all oscillators on both sides contribute to the swing. Near the endpoints, there are fewer assisting oscillators outside. Therefore, the loads on these oscillators are higher than in the middle. They are still synchronized but lag behind. The skew compared to the middle oscillator has been simulated for vectors that are 25 and 64 elements long, to $21 \mathrm{ps}$ and 35 ps respectively, see Fig. 8. Inside the fourth oscillator from the endpoint, the skew between adjacent oscillators is less than $2 \mathrm{ps}$. At the outermost point, the skew is $7 \mathrm{ps}$.

The clock skew can easily almost be eliminated by adjusting the free running frequencies of the oscillators with strategically selected capacitances. If $0.33 \mathrm{fF}$ and $0.24 \mathrm{fF}$ are added to the second and third oscillators from the endpoint, and $0.22 \mathrm{fF}$ for those inside, while the outermost is uninfluenced in the 25 elements long vector, the total skew will be less than $3.3 \mathrm{ps}$, see Fig. 8. The maximum skew of 1.2 ps is between the two outermost oscillators. However, this is not planned to be used in the SBP. Instead, the skew is controlled for low power noise, see section VII.
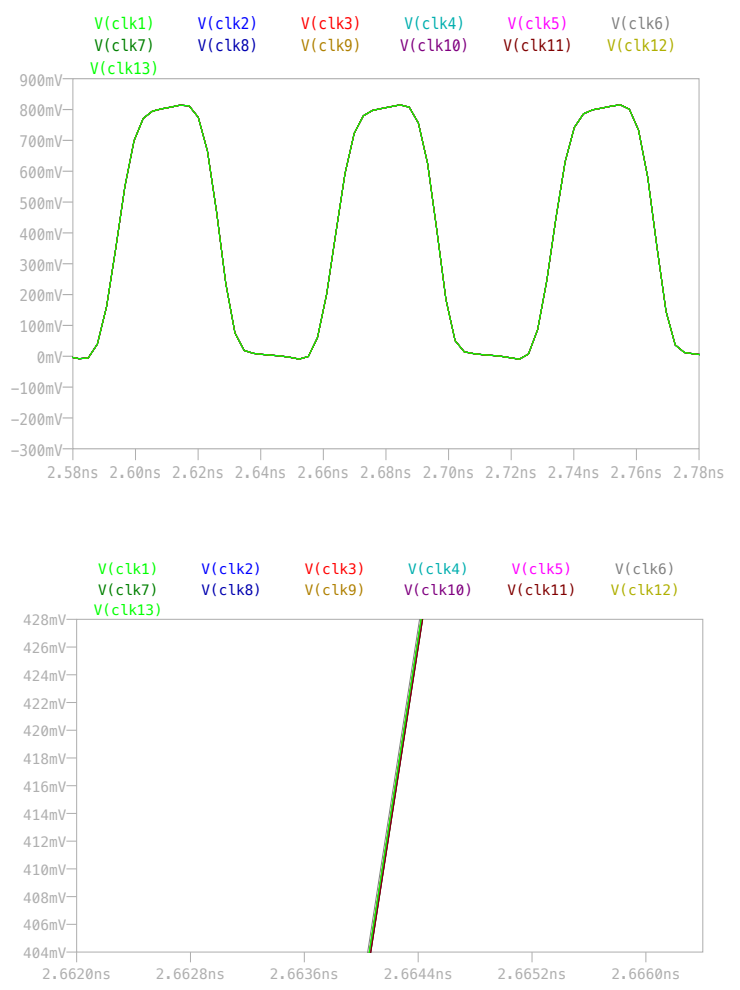

Fig. 7. Clock skew in a loop of 25 and 64 oscillators (top view) where the individual signals are indistinguishable, with a magnification of a slope.
To further verify the theory, a mesh of arrays instead of vectors has been simulated. A network with $200 \mu \mathrm{m}$ grid was used and the simulations were run with an odd number of elements in the array, from $3 \times 3$ up to $25 \times 25$. They all behaved in the same way. When using a short simulation time, it was apparent that there was a kind of wave-like pattern flowing through the array. We conclude that this is caused by feedback from the edges. The situation became stable within $10 \mathrm{~ns}$. We also simulated a $33 \times 33$ array for $10 \mathrm{~ns}$. With a 12 -core CPU, this processing took more than a week. This array did not stabilize within the $10 \mathrm{~ns}$ time frame. The time to reach a steady state increases with the array size.

Fig. 9 shows the result for an array of $25 \times 25$ oscillators. They are enumerated sequentially, from left to right, and from top to bottom. The skew is shown along the vertical axis and the oscillator number on the horizontal axis. Each of the $25 \mathrm{U}-$ shaped segments shows the skew along one row.

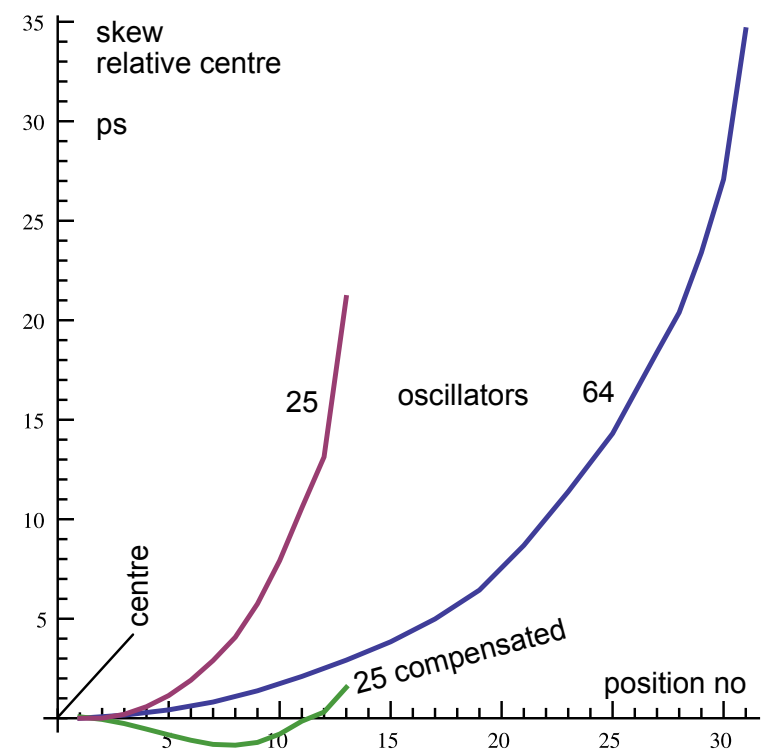

Fig. 8. Clock skew in a vector with 25 and 64 oscillators, and with adjustment from two capacitors added for 25 elements.

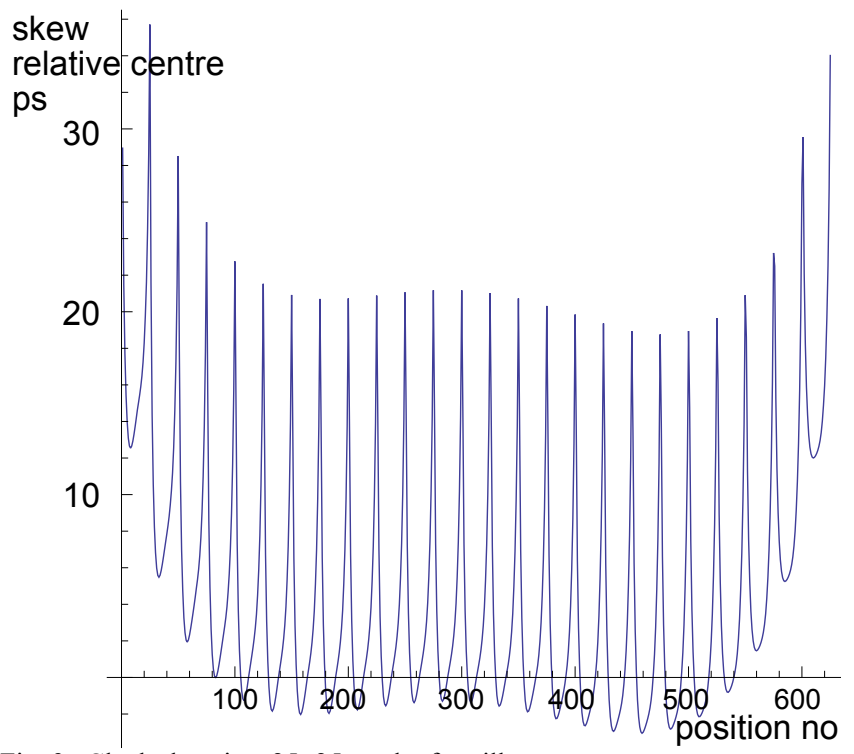

Fig. 9. Clock skew in a $25 \times 25$ mesh of oscillators. 


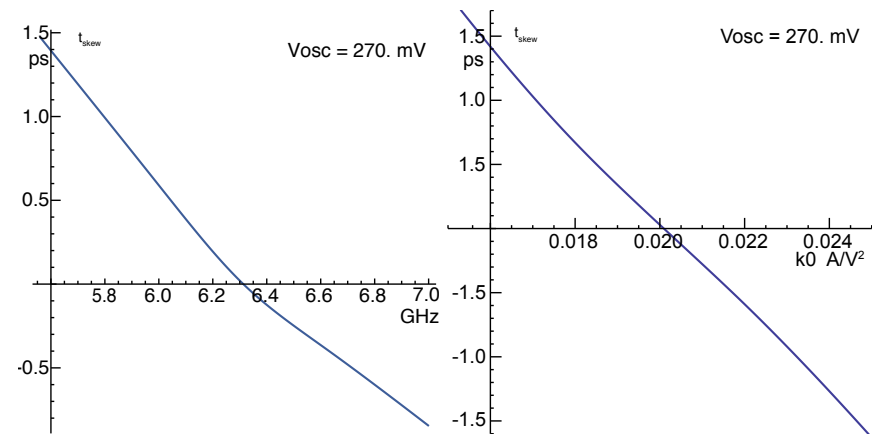

Fig. 10a. The oscillator skew dependency of free running frequency.

Fig. 10b. The oscillator skew dependency of the clock bus current.

\section{PROCESS VARIATIONS}

The oscillators and the pulse shaping devices differ somewhat due to process variations, which make the free running clock frequencies vary. An oscillator with a slightly higher frequency will have a skew before a slower one. Differences in the thresholds of the pulse shaping devices also cause skew.

\section{A. Clock Skew}

The clock skew was analyzed by simulating a triangle with identical $200 \mu \mathrm{m}$ long wires. The components have been assumed to possess nominal properties, and random deviations have not been taken into account.

Two of the oscillators are equal and have nominal characteristics. In the third oscillator, the characteristics are altered by changing the design parameters. The supply voltage $V_{\text {osc }}$ is fixed, while the free running frequency is varied between 5.56 and $7.0 \mathrm{GHz}$, i.e., \pm 12 percent, by changing a capacitance in the first stage, see Fig. 10a. The PE-internal clock skew is within the range 1.47 to -0.84 ps. In another simulation, the current fed into the clock bus is altered by changing the Spice parameter $K p$ in the range 15 to $25 \mathrm{~mA} / \mathrm{V}^{2}$, i.e., \pm 14 percent, see Fig. 10b. The clock skew is then within the range 1.83 to $-1.63 \mathrm{ps}$.

\section{B. Pulse Shaper Skew}

The pulse shaper was analyzed by changing the design parameters. The threshold voltage of transistors $M 8$ and $M 10$ is varied between 30 and $90 \mathrm{mV}$, see Fig. 11a. The PEinternal clock skew is within the range -4.2 to -2.9 ps. In another measurement, the current is varied by the Spice parameter $K p$ between 1.8 and $3.0 \mathrm{~mA} / \mathrm{V}^{2}$, see Fig. $11 \mathrm{~b}$. The clock skew is then within the range -2.4 to $2.1 \mathrm{ps}$.

\section{PE Skew}

The nominal clock skew in relation to the internal clock $C L K$ is positioned within a 7.4-14.9 ps window. The skew between internal registers is somewhere between -0.9 and +1.5 ps. The computing area of the SBP where arithmetic is performed is about $10 \times 200 \mu \mathrm{m}^{2}$. Here, the skew increases the propagation delay.

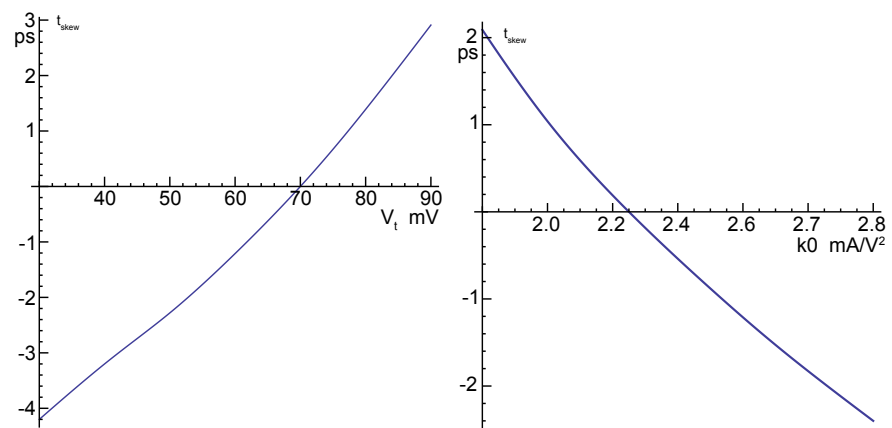

Fig. 11a. The pulse shaper skew dependency of transistor threshold voltage. Fig. 11b. The pulse shaper skew dependency of transistor current.

\section{REDUCing Power NoISE By SKEWING OSCILlators}

A clock transition causes switching of clock buffers and registers. This triggers events on wires, which switch gates. High currents charge wires from Vdd and discharge them to ground. Additional peak currents originate from short circuiting during transitions of gate voltages. If many wires switch simultaneously, the supply voltage will ripple considerably.

A large portion of the current is used to charge long wires in memories and buses. In SBP, each PE is a clock region, where the clock skew is very low. The array of PEs can, on the other hand, have a gradual skew along an axis of the grid since all communication is between adjacent PEs and a very small controlled skew between neighboring PEs along one axis will not affect the performance. The skew should be slightly less than one clock cycle between the edges of the array. The aggregate supply current will be an average for a whole clock cycle. It ripples depending on the local execution in all PEs, however, instantaneously from only some few PEs and with a much higher frequency.

The skew is accomplished by gradually increasing the frequency of free running oscillators. This is achieved by raising the capacitances of the oscillators. The skews for a vector of oscillators where the capacitance is increased by $35 \mathrm{aF}$ between each is shown in Fig. 12.

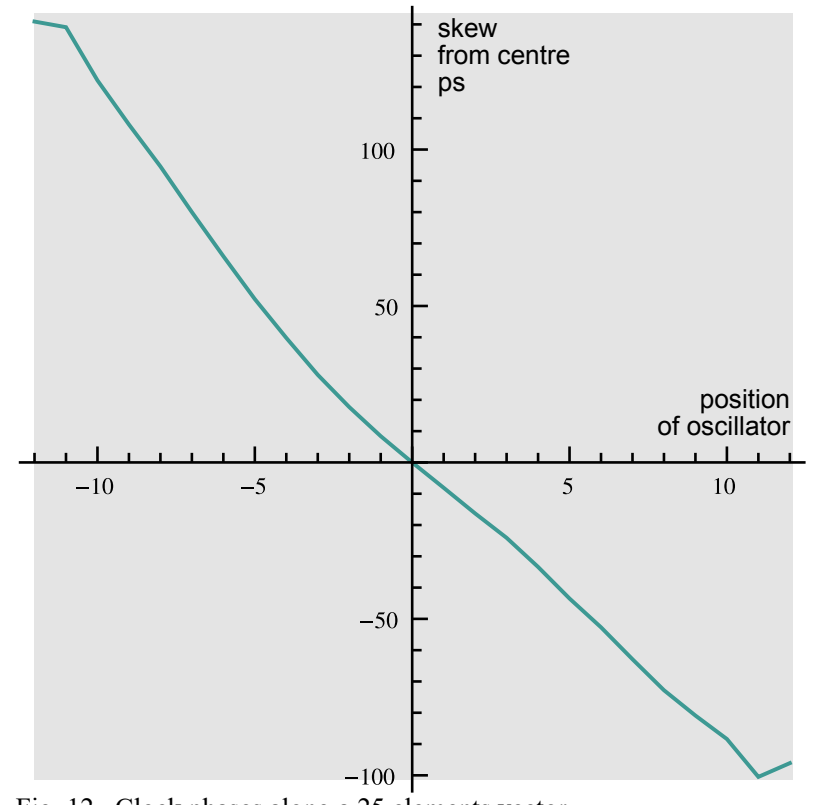

Fig. 12. Clock phases along a 25 elements vector. 


\section{RELATED WORK}

A literature search concerning clocking has been conducted using the IEEE Xplore database, and 561 papers were found. Most of them concerned placement and synthesis for H-tree shaped clock distributions with optimized wire widths, the placement of buffers, and their driving capacity. No paper was found that focused on high frequency. Also, no papers seemed to focus on large meshes of clock regions with PEs.

\section{A. Transmission Lines}

Transmission lines and negative resistance in loops may be used as oscillators. A mesh of transmission lines with negative resistance in the nodes may contain many oscillators. The oscillators have the same frequency and low skew [3], [4], [5]. The synchronization algorithm is the same as presented here. However, they used transmission lines with inductances, which are outdated since around 2005.

Strongly coupled RC-oscillators will lock their frequencies and acquire almost the same phase when connected through nearly lossless transmission lines [6] (in 1996) and [7] (in 1998). A grid or mesh may comprise many rings shaped by directed links, constituting delay elements. In each node, there is a summation and inversion. The entire structure will oscillate [8] (in 1997).

\section{B. Synchronization}

A clock system based on a mesh of regions where the oscillators are based on phase locked loops is presented in [9]. Between each region in both directions there is an analogue phase detector. The phase error is summed up in an amplifier that controls the frequency of an oscillator. The synchronization signals have low voltage. The aim and principle are the same as presented here, but more complex. A system of $4 \times 4$ oscillators was analyzed, where the clock period was $830 \mathrm{ps}$ and the clock jitter 3.6 percent. The clock skew was not presented. The power consumption was $24 \mathrm{~mW}$ per oscillator in $0.35 \mu \mathrm{m}$ technology.

\section{Buffers}

Clock buffers without short circuit current have been presented [10], where a third transistor is inserted between the $\mathrm{n}$ - and $\mathrm{p}$-transistors of an inverter. It isolates during the transitions, when feedback from the output prevents conductivity. The switching current is reduced to half during the falling transitions. Therefore, two transistors occupying twice the space are needed.

\section{Power Consumption}

The power consumption of a circuit depends on a lot of things, and it is almost impossible to give an appropriate measure. However, the effective sink load may be one way to put it: the quotient of an effective capacitance $\mathrm{C}_{\text {eff }}$ to the standard gate capacitance $\mathrm{Cg}$. The power of a circuit is converted to the effective switching energy per sink gate (not full circuit). The energy is converted to a corresponding capacitance that dissipates the same energy when dividing it by $\mathrm{Vdd}^{2} / 2$. This measure is mostly technology independent.
The power consumption presented in this article, $807 \mu \mathrm{W}$ per PE with 93 registers each containing $4+4$ gates, see section $I V . A$, corresponds to $1.08 \mu \mathrm{W}$ per gate. At $6.7 \mathrm{GHz}$, the switching energy is $163 \mathrm{aJ}$ per gate. At $0.8 \mathrm{~V}$, this corresponds to a capacitance of $509 \mathrm{aF}$, or 7.3 gate capacitances.

The energy stored in a capacitor is $\mathrm{C} \times \mathrm{V}_{\mathrm{c}}{ }^{2} / 2$, while the energy supplied from a power source is $\mathrm{C} \times \mathrm{Vdd} \times \mathrm{V}_{c}$, i.e., proportional to the supply voltage Vdd and the voltage swing $\mathrm{V}_{\mathrm{c}}$, and in this case $\mathrm{Vdd}=\mathrm{V}_{\mathrm{c}}$. Therefore, 2 gate capacitances are the basic level. The additional $2 \times 2.65$ times to reach 7.3 are required for short circuit currents and wiring.

A corresponding power figure is very hard to find in a literature search. Generally, at least one parameter is missing.

Two competitions were held on the synthesis of clock networks in the International Symposium on Physical Design (ISPD) 2009 [11] and 2010 [12], and we have compared our work to the results of the first benchmark suite. Since the benchmarks were not technology independent, the $45 \mathrm{~nm}$ technology used back then has been measured against the $14 \mathrm{~nm}$ in this work, implying a threefold improvement may be expected.

There were two measured results: clock skew and power consumption. The evaluation of the clock skew had some simplifications and used a netlist very different from ours, but the results were overall at least one order of magnitude worse than we have presented.

The power consumption can be expressed less technology dependent. In 2010, the rules were actually revised, and the clock power modeled by capacitance was the major judging criteria, but this contest was not at all described in as much detail as the one in 2009. The criteria in 2009 was to keep within a power envelope specified for each test while minimizing the skew. The winners generally opted to use as much as possible of the available power, which in most cases was around 30 times the number of sinks. This value may possibly be compared to 7.3 , presented in this article, but with a much smaller skew in the implementation described here.

\section{CONCLUSION}

A clock system based on synchronized oscillators has been presented, where the synchronization comes from a mesh of wires. The clock skews are zero for an infinite network, which has been stated in section II and shown in section $V$ by connecting a sequence of oscillators into a loop. A finite network has fringe effects, where the clock skew between two neighboring oscillators is low and increases towards the edges. Uncompensated grids of $3 \times 3$ to $25 \times 25$ oscillators have been simulated and show the same fringe effect in two dimensions. By adjusting the free running frequencies of the oscillators, the maximum skew between two adjacent oscillators can be reduced to $1.2 \mathrm{ps}$, as was also demonstrated in section $V$.

Oscillators with a sinusoidal output of $200 \mathrm{mV}$ are used in the clock system. This signal is converted to a square wave in a pulse shaping stage, as can be seen in section III. 
The system of oscillators is used to demonstrate the high frequency properties for a set of PEs in section $I V$. The PEs are from a design with 93 master-slave flip-flops and has an aggregated clock load of $83 \mathrm{fF}$. A clock buffer is used to drive a T-shaped structure of $80 \mu \mathrm{m}$ clock wires in $14 \mathrm{~nm}$ technology. The clock buffer consists of a single inverter with small dimensions, supported by one current surge driver for each polarity in order to avoid short circuit current. The clock wire drives 19 second-level buffers. The clock skew is within $2.3 \mathrm{ps}$ between the registers at $6.7 \mathrm{GHz}$. The power consumption is $807 \mu \mathrm{W}$ per PE, corresponding to an effective switching gate capacitance of $509 \mathrm{aF}$ or 7.3 gate capacitances.

Power noise can be reduced by scheduling the clocks, as was shown in section VII. This scheduling is preferably performed between PEs, and not within them. It can be accomplished by adjusting the oscillators to have a slight intentional skew, by using a small capacitive load in the oscillators to give them a gradually increasing free running clock speed.

Only local process variations influence the clock skew. These variations are negligible within a clock region, as was shown in section VI.

The ambition to design an optimal clock system for an SBP has been fulfilled. It can also be used to improve other multicore and manycore systems.

\section{ACKNOWLEDGMENT}

The authors wish to thank Prof. Christer Svensson at Linköping University for his valuable and constructive comments. We also appreciate the support from Prof. Per Stenström at Chalmers University of Technology.

\section{REFERENCES}

[1] M. Rimborg, P. Trancoso and G. Carlstedt, "PHOENIX: Efficient Computation in Memory," Proceedings of the International Symposium on Memory Systems, Alexandria, VA, October 2017, pp. 15-25, https://doi.org/10.1145/3132402.3132430.

[2] E. G. Friedman, "High Performance Clock Distribution Networks," in: E. G. Friedman, High Performance Clock Distribution Networks, Springer, Boston, MA, USA, 1997, pp 1-4.

[3] J. Wood, T. C. Edwards and S. Lipa, "Rotary traveling-wave oscillator arrays: a new clock technology," in IEEE Journal of Solid-State Circuits, vol. 36, no. 11, Nov. 2001, pp. 1654-1665.

[4] J. Wood, S. Lipa, P. Franzon and M. Steer, "Multi-gigahertz low-power low-skew rotary clock scheme," 2001 IEEE International Solid-State Circuits Conference. Digest of Technical Papers. ISSCC (Cat. No.01CH37177), San Francisco, CA, USA, 2001, pp. 400-401.

[5] S. C. Chan, K. L. Shepard and P. J. Restle, "Design of resonant global clock distributions," Proceedings 21st International Conference on Computer Design, San Jose, CA, USA, 2003, pp. 248-253.

[6] I. Galton, D. A. Towne, J. J. Rosenberg and H. T. Jensen, "Clock distribution using coupled oscillators," 1996 IEEE International Symposium on Circuits and Systems. Circuits and Systems Connecting the World. ISCAS 96, Atlanta, GA, USA, 1996, pp. 217-220 vol. 3.

[7] H. Mizuno and K. Ishibashi, "A noise-immune GHz-clock distribution scheme using synchronous distributed oscillators," 1998 IEEE International Solid-State Circuits Conference. Digest of Technical Papers, ISSCC. First Edition (Cat. No.98CH36156), San Francisco, CA, USA, 1998, pp. 404-405.

[8] L. Hall, M. Clements, Wentai Liu and G. Bilbro, "Clock distribution using cooperative ring oscillators," Proceedings Seventeenth Conference on Advanced Research in VLSI, Ann Arbor, MI, USA, 1997, pp. 62-75.

[9] V. Gutnik and A. P. Chandrakasan, "Active GHz clock network using distributed PLLs," in IEEE Journal of Solid-State Circuits, vol. 35, no. 11, Nov. 2000, pp. 1553-1560.

[10] S. Shim, M. Mo, S. Kim and Y. Shin, "Analysis and minimization of short-circuit current in mesh clock network," 2013 IEEE 31st International Conference on Computer Design (ICCD), Asheville, NC, 2013, pp. 459-462

[11] C. N. Sze, P. Restle, G.-J. Nam and C. Alpert, "Ispd2009 clock network synthesis contest," Proceedings of the 2009 international symposium on Physical design (ISPD '09), Association for Computing Machinery, New York, NY, 2009, pp. 149-150.

[12] C. N. Sze. 2010, "ISPD 2010 high performance clock network synthesis contest: benchmark suite and results," in Proceedings of the 19th international symposium on Physical design (ISPD '10), Association for Computing Machinery, New York, NY, 2010, p. 143. 